\title{
Quantification of flows generated by the hydromedusa Aequorea victoria: a Lagrangian coherent structure analysis
}

\author{
Kakani Katija ${ }^{1,4, *}$, Wesley T. Beaulieu ${ }^{2}$, Charlotte Regula $^{3}$, Sean P. Colin ${ }^{3}$, \\ John H. Costello ${ }^{2}$, John O. Dabiri ${ }^{1}$ \\ ${ }^{1}$ Graduate Aeronautical Laboratories and Bioengineering, California Institute of Technology, Pasadena, California 91125, USA \\ ${ }^{2}$ Biology, Providence College, Providence, Rhode Island 02918, USA \\ ${ }^{3}$ Biology and Marine Biology, Roger Williams University, Bristol, Rhode Island 02809, USA \\ ${ }^{4}$ Present address: Applied Ocean Physics and Engineering, Woods Hole Oceanographic Institution, Woods Hole, \\ Massachusetts 02543, USA
}

\begin{abstract}
Most oblate medusae use flow generated during swimming to capture prey. Quantification of their interactions with surrounding fluid is necessary to understand their feeding mechanics and to develop models to predict their predatory impact. In the present study, we quantified how the hydromedusa Aequorea victoria interacts with both its surrounding fluid and prey. The fluid interactions were examined in the laboratory and in natural field settings using digital particle image velocimetry (DPIV) measurements. The laboratory DPIV data were used to compute finite-time Lyapunov exponent (FTLE) fields, and Lagrangian coherent structures (LCS) were extracted from the FTLE fields. The laboratory LCS analysis demonstrated that swimming A. victoria only encounter discrete packets of fluid originating upstream of the medusan bell. Based on the size of these packets, we estimated that the $A$. victoria examined have the potential to clear $11.4 \mathrm{l} \mathrm{h}^{-1}$. Used in conjunction with measured prey capture efficiencies, we estimated potential clearance rates on different prey types. These hydrodynamically based clearance rate estimates are consistent with previously measured empirical clearance rate estimates. Velocity vector and shear fields also suggested that the feeding current created by $A$. victoria may be more suitable for encountering copepods than previously thought. Although still preliminary, in situ DPIV data indicate that natural background flows may alter the encounter process from what is observed in still-water laboratory conditions.
\end{abstract}

KEY WORDS: Lagrangian coherent structures $\cdot$ Medusae $\cdot$ Field measurements $\cdot$ Digital particle image velocimetry $\cdot$ Predator-prey interactions

\section{INTRODUCTION}

Medusae are known to be voracious predators capable of impacting pelagic communities (Matsakis \& Conover 1991). Most species capable of exerting a high predatory impact forage as feeding current predators (Costello et al. 2008 and references therein). Feeding current-dependent foragers are characterized by nonsaturating ingestion rates, even under very high prey concentrations (Costello \& Colin 1995, Purcell \& Cowan 1995, Titelman \& Hansson 2005). These feeding mech- anics, coupled with often high population densities of the medusae, are critical factors determining the important role played by these medusan predators in planktonic communities. The feeding currents generated by these predators are produced during medusan swimming (Mills 1981, Costello \& Colin 1994, 1995), and therefore it is important to quantitatively evaluate the role of fluid interactions between swimming medusae and their prey.

Swimming by medusae depends upon rhythmic contraction and relaxation of their swimming bells. In 
addition to providing thrust, these bell motions transport fluid to prey capture surfaces (i.e. tentacles and manubrium-oral arms), where entrained prey may be retained (Costello \& Colin 1995, Colin et al. 2006, Peng \& Dabiri 2009). The fluid interactions and patterns of flow around swimming-cruising medusae have been well described qualitatively (Dabiri et al. 2005, Colin et al. 2006). These studies have shown that as the bell expands, fluid adjacent to the bell margin is entrained and drawn through trailing tentacles into a stopping vortex ring (structure created at the end of a swimming cycle) that rotates inside the subumbrellar cavity. As the bell contracts, fluid continues to be entrained adjacent to the bell margin and, along with fluid from the stopping vortex, is drawn into a starting vortex ring (structure created at the start of a swimming cycle) that rotates through the trailing tentacles in the wake of the medusa. Consequently, fluid appears to be continuously entrained throughout the swimming cycle and interacts with capture surfaces (Dabiri et al. 2005).

While these studies have provided an understanding of the general nature of fluid transport around the bell and in the medusan wake, they have not characterized the volume flux of fluid around the bell during swimming (or encounter volume rate, $E$ ). Few studies have attempted to quantify the flow around medusae (Shadden et al. 2006, Peng \& Dabiri 2009), yet modeling and prediction of ingestion and prey selection by cruising medusae requires a quantitative description of how these medusae entrain fluid while swimming. Characteristics of fluid transport affect feeding by determining the amount and types of prey entrained and encountered. Encounter rates (defined here as the number of prey that enter the capture zone over time) are controlled by the amount of fluid that is entrained and transported past capture surfaces over time. The type and amount of prey entrained will largely be determined by prey characteristics such as size, swimming speed and reactivity (Gerritsen \& Strickler 1977, Suchman \& Sullivan 1998, Viitasalo et al. 1998) in relation to fluid characteristics such as flow velocities and deformation rates (Ford et al. 1997, Kiørboe et al. 1999, Buskey et al. 2002). These important fluid parameters have yet to be quantified in detail for medusae.

Quantitative studies of flow surrounding swimming animals have been conducted in the past and have utilized techniques that include digital particle image velocimetry (DPIV; Videler et al. 2002, Tytell \& Lauder 2004). Laboratory settings are necessary for pragmatic reasons and enable high-quality detailed descriptions of animal-fluid interactions. However, the relevance of these descriptions in natural field conditions is generally unknown. For medusae, qualitative flow visualizations conducted in the laboratory that identified important fluid structures around medusae (e.g. stopping and starting vortices; Costello \& Colin 1995, Colin \& Costello 2002, Raskoff 2002) have been shown to be relevant in the field (Dabiri et al. 2005, Colin et al. 2006). However, the quantitative characteristics of flow around medusae are likely to be altered by natural flow and turbulence conditions (Kiørboe et al. 1999). For this reason, it is important to develop approaches that can be used to evaluate impacts of natural flow patterns on the fluid processes governing predatorprey interactions.

Our approach towards reaching this goal was to use a new analysis tool called Lagrangian coherent structures (LCS) derived from DPIV data (Haller 2001, Shadden et al. 2005, 2006) to quantify the fluid interactions of swimming medusae. While conventional DPIV analysis computes velocity vector fields that can be used to estimate fluid energetics and identify fluid structures such as vortex rings (Shadden et al. 2006), LCS analysis expands the utility of conventional DPIV by increasing our ability to describe and quantify feeding-related fluid transport around swimming medusae. Here we describe these patterns for the feeding current-foraging leptomedusa Aequorea victoria. Members of the genus Aequorea are distributed throughout the world's oceans and have been demonstrated to be influential predators on a range of zooplankton, including fish eggs and larvae (Purcell 1989, 1991, Costello \& Colin 2002). The recent extension of DPIV techniques from the laboratory to in situ conditions using a selfcontained underwater velocimetry apparatus (SCUVA Katija \& Dabiri 2008) enabled us to compare the laboratory results with a field data set.

\section{MATERIALS AND METHODS}

Experimental method. Measurements of capture efficiency were conducted at the Marine Biological Laboratory (Woods Hole, Massachusetts). Aequorea victoria specimens, approximately $5 \mathrm{~cm}$ in diameter, were acquired from the New England Aquarium (Boston, Massachusetts) and held in containers for acclimation to room temperature before measurements. Four species of prey were used in the present study: the brine shrimp Artemia salina, adult Acartia tonsa copepods, juvenile Mnemiopsis leidyi ctenophores, and the eggs of Libinia emarginata crabs. These prey were chosen to compare the effects of hard- (Ar. salina and Ac. tonsa) or soft-bodied prey (L. emarginata and M. leidyi), prey size and escape behavior on capture by Ae. victoria. Ar. salina were cultured in the laboratory from dehydrated cysts (Connecticut Valley Biological Supply) and Ac. tonsa and M. leidyi were collected in Woods Hole using standard plankton net tows. Eggs of $L$. emarginata were harvested from live females collected 
off the coast of Woods Hole. Rectangular filming vessels were filled with filtered seawater. Medusae were placed in the vessel with a monoculture of prey. We analyzed video only when the tentacles of $A$. victoria were extended. The tentacles were never extended longer than 1 or 2 bell diameters behind the medusa. To analyze capture efficiencies (see Fig. 3), medusae and prey were filmed and efficiencies quantified from $30 \mathrm{~h}$ of video with 23 medusae, yielding 214 encounters. Encounters (defined as an event in which a prey individual is entrained in the medusa's wake and passes through its tentacles) and captures (an event in which a prey is encountered and adheres to a medusa's tentacles for $>2$ s) were quantified from the video footage. Finally, the capture efficiency (a) was determined by the ratio between the number of prey captured and the number of prey encountered.

Quantitative flow measurements in the laboratory and in situ were conducted at Friday Harbor Laboratories in Friday Harbor, Washington. Animals were collected off the Friday Harbor Laboratory dock and acclimated to room temperature before conducting laboratory measurements. In the laboratory, Aequorea victoria specimens, $\sim 5 \mathrm{~cm}$ in diameter, were placed in large glass filming vessels $(35 \times 15 \times 35 \mathrm{~cm}$; length $\times$ depth $\times$ height). These dimensions were several body lengths greater than the medusa in order to minimize vessel artifacts in the flow. The aquarium was filled with natural, unfiltered seawater that contained sufficient suspended particulates to enable quantitative visualization. Fluorescent dye was used to qualitatively describe the fluid structures generated by a swimming animal. The flow field was quantitatively measured in the laboratory and in situ using DPIV (Adrian 1991, Willert \& Gharib 1991, Dabiri et al. 2010). Components for DPIV experiments included a laser (for flow illumination), optics (designed to spread the laser beam into a thin sheet to illuminate a plane in the flow), a camera to record consecutive images, and a software package that uses a cross-correlation scheme to analyze particle displacements and output velocity fields. The flow field was illuminated by a single $350 \mathrm{~mW}, 532 \mathrm{~nm}$ solid-state laser (Wicked Lasers), with optics that created a sheet $<2 \mathrm{~mm}$ thick. A video camera (Sony HDR-FX1) was oriented perpendicular to the light sheet and captured images in the laser plane at $700 \times 480$ pixel resolution and 30 frames $\mathbf{s}^{-1}$. Measurements in the natural field setting were accomplished by using SCUVA (Katija \& Dabiri 2008), which used the same laser and video camera as described for the laboratory. Only sequences in which the laser sheet remained in the center of the medusa were used for analysis. Laser light sheet alignment through the center of the animal body was identified when the manubrium (centrally located in the subumbrellar cavity) was fully illuminated.
In the laboratory, 5 Aequorea victoria were videotaped while swimming for multiple pulse cycles through the laser-illuminated field of view. Additionally, a single data set with multiple swimming cycles (necessary for the LCS analysis) was obtained in situ. Based on the location of the laser sheet relative to the camera, a conversion ratio of 26 pixels $\mathrm{cm}^{-1}$ (laboratory) and 45 pixels $\mathrm{cm}^{-1}$ (in situ) was used for subsequent DPIV analysis. Each image was evaluated with an interrogation window size of $16 \times 16$ pixels and a $50 \%$ overlap. Calculations of velocity and vorticity fields reflect an uncertainty of 1 and $3 \%$, respectively (Dabiri \& Gharib 2004). The DPIV analysis produced velocity field data every $0.033 \mathrm{~s}$ over a domain approximately $18 \times 28 \mathrm{~cm}^{2}$ in the laboratory and approximately $10 \times 16 \mathrm{~cm}^{2}$ in the field. These velocity data were used to compute the flow map for the LCS analysis. For this experiment, long integration time $T$ corresponds to 60 frames of data (or $|T|=60$ frames) and short integration times correspond to $|T|=30$ frames. Velocity vectors for the in situ data have been corrected for diver motion by subtracting the mean flow, which is computed by averaging the components of velocity in the $x$ - and $y$-directions from the entire velocity field (cf. Katija \& Dabiri 2008). Maximum animal diameter and average distance traveled per swimming cycle were determined from consecutive video images. To avoid erroneous vectors due to animal body motion, the body outline was identified by thresholding the intensity of the images; any vectors located within the animal body were set to zero. The twodimensional vorticity $(\omega)$ and total shear $(\tau)$ were found directly from DPIV velocity fields using the methods of Batchelor (1967) such that:

$$
\begin{gathered}
\omega=\frac{\mathrm{d} v}{\mathrm{~d} x}-\frac{\mathrm{d} u}{\mathrm{~d} y} \\
\tau=\frac{\mathrm{d} u}{\mathrm{~d} y}+\frac{\mathrm{d} v}{\mathrm{~d} x}
\end{gathered}
$$

Lagrangian method. LCS are boundaries in a fluid that distinguish regions of differing dynamics where maximum separation between initially adjacent parcels of fluid occurs. For this reason, LCS are used to identify regions of laminar flow from turbulence (i.e. Green et al. 2007), vortex rings (Romkedar et al. 1990, Shadden et al. 2006), and animal wakes (i.e. Shadden et al. 2006, Peng \& Dabiri 2007). Therefore, we propose that LCS can be used to indicate boundaries between regions of flow adjacent to a swimming animal and its wake structures from the surrounding background flow.

Because maximum divergence of fluid parcels will indicate LCS, the objective of this analysis is to find a measure of maximum fluid separation over some integration time during which the flow is observed. Since fluid separation is experimentally determined by the 


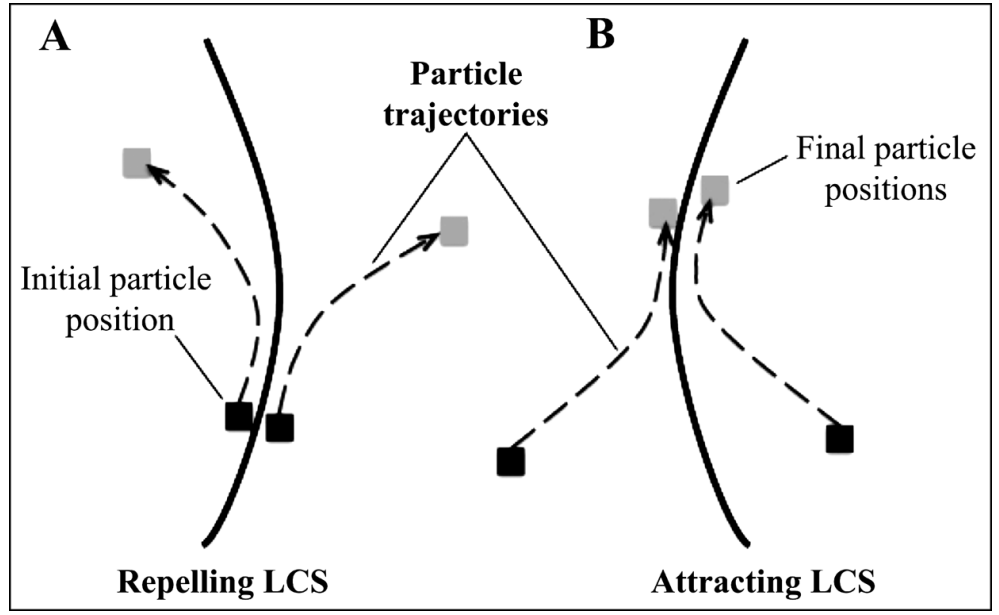

Fig. 1. Behavior of particles near Lagrangian coherent structure (LCS) curves (solid lines). As time progresses forward, the position of 2 initially adjacent particles on either side of a repelling LCS diverge (Panel A). For 2 particles initially on either side of an attracting LCS (Panel B), as time progresses forward their positions converge on the attracting LCS curve. Dashed gray curves: particle trajectories with arrows indicating direction; dark and light gray boxes: initial and final particle positions, respectively

displacement of particles suspended in a fluid using DPIV, we will use particle separation as a proxy for fluid separation in the measurement domain (Fig. 1).

The quantity that measures particle separation is called the finite-time Lyapunov exponent (FTLE; Shadden et al. 2005) or direct Lyapunov exponent (Haller 2001). Given an empirically produced flow (i.e. from experiments or numerical simulations), the displacement of adjacent particles at time $t_{0}$ [initially separated by a distance $\left.\delta x\left(t_{0}\right)\right]$ and the displacement after some integration time $T$ of the same particle pair [denoted as $\left.\delta x\left(t_{0}+T\right)\right]$, the finite-time Lyapunov exponent $(\sigma)$ can be defined as:

$$
\sigma_{t_{0}}^{T}(x)=\frac{1}{|T|} \ln \left\|\frac{\delta x\left(t_{0}+T\right)}{\delta x\left(t_{0}\right)}\right\|
$$

The FTLE is evaluated for each node of the FLTE grid within the measurement domain, and results in a scalar field. Smaller FTLE values indicate regions in the flow that lack strong fluid parcel separation, larger values of FTLE indicate regions with higher fluid parcel separation. Once the FTLE field is found, the LCS can be determined from the hyperbolic 'ridges' or local maxima of FTLE in the flow (Haller 2001, 2002, Shadden et al. 2005, Mathur et al. 2007).

For example, consider the black curves in Fig. 1, which correspond to LCS with differing behaviors. Two particles are initially close together and are located on either side of the LCS curve (Fig. 1A). As time proceeds forward, these 2 particles remain on either side of the LCS and their particle paths diverge (dashed, gray curves). This LCS, which is found by using a positive integration time $(T>0$ or forward-time), is formally called the repelling LCS. A second type of LCS can be found by using a negative integration time ( $T<0$ or backward-time); these are attracting LCS. Two particles are initially far from each other and are located on either side of an attracting LCS (Fig. 1B). As time continues, the paths of the same particle pair converge. By combining the forward- and backward-time, or repelling and attracting LCS curves, structures such as vortex rings and recirculation regions can be identified (Romkedar et al. 1990, Shadden et al. 2006, Franco et al. 2007). Larger integration time $T$ increases spatial resolution of the LCS; however, the choice of $T$ is limited by the availability of data and computational costs.

In studies utilizing the LCS analysis, upstream LCS lobes identify regions in the fluid that become entrained by swimming medusae (Shadden et al. 2006, Peng \& Dabiri 2009). Therefore, by measuring the shape and size of LCS lobes upstream of a swimming animal, we can quantify the volume of fluid that will be encountered by its tentacles. Consequently, since each upstream LCS lobe corresponds to a single swimming cycle, the volume of an LCS lobe indicates the volume of fluid entrained by the animal per swimming cycle. We defined entrained fluid (i.e. encountered fluid) as the fluid that is transported by bell motions along to the bell margin and into the region just posterior to the bell within the diameter of the bell. This is the region where the tentacles are commonly positioned and through which fluid is primarily entrained and encountered by oblate rowing medusae (Costello \& Colin 1994, 1995, Dabiri et al. 2005). However, based on this definition of encounter zone, our estimates should represent conservative estimates of encounter rates, since the tentacles often extend beyond this region and we did not consider fluid that passes through the tentacles further downstream. Since the LCS analysis yields lobe areas (from a 2-dimensional data set), the fluid entrainment volume per pulse is found by assuming radial symmetry of the upstream LCS lobes and revolving the area about the animal body's axis. Clearance rates were determined from the ratio of upstream LCS lobe volume and duration of a swimming cycle. Laboratory results summarized in Table 1 are based on 5 different medusae with multiple swimming cycles (each medusa constituting a different data set); in situ values are based on a single medusa with 3 consecutive swimming cycles. All the analyses requiring the quantification of LCS utilized 
Table 1. Summary of relevant Lagrangian coherent structure (LCS) parameters from digital particle image velocimetry (DPIV) measurements of Aequorea victoria swimming in the laboratory (mean $\left.\pm \mathrm{SD}_{i} \mathrm{n}=5\right)$ and in situ $(\mathrm{n}=1)$

\begin{tabular}{|lccccccc|}
\hline & $\begin{array}{c}\text { Max. } \\
\text { animal } \\
\text { diameter }(\mathrm{cm})\end{array}$ & $\begin{array}{c}\text { Max. } \\
\text { marginal } \\
\text { velocity }\left(\mathrm{cm} \mathrm{s}^{-1}\right)\end{array}$ & $\begin{array}{c}\text { Max. } \\
\text { marginal } \\
\text { vorticity }\left(\mathrm{s}^{-1}\right)\end{array}$ & $\begin{array}{c}\text { Max. } \\
\text { marginal } \\
\text { shear }\left(\mathrm{s}^{-1}\right)\end{array}$ & $\begin{array}{c}\text { Upstream } \\
\text { LCS lobe } \\
\text { area }\left(\mathrm{cm}^{2}\right)\end{array}$ & $\begin{array}{c}\text { Upstream } \\
\text { LCS lobe } \\
\text { volume }\left(\mathrm{cm}^{3}\right)\end{array}$ & $\begin{array}{c}\text { Encounter } \\
\text { volume } \\
\text { rate, } E\left(\mathrm{~h}^{-1}\right)\end{array}$ \\
\hline $\begin{array}{l}\text { In vitro } \\
\text { In situ }\end{array}$ & $\begin{array}{c}5.56 \pm 0.29 \\
2.78\end{array}$ & $\begin{array}{c}6.12 \pm 0.62 \\
0.73\end{array}$ & $\begin{array}{c}0.37 \pm 0.04 \\
0.44\end{array}$ & $\begin{array}{c}0.50 \pm 0.02 \\
2.38\end{array}$ & $\begin{array}{c}1.22 \pm 0.20 \\
0.42\end{array}$ & $\begin{array}{c}21.96 \pm 2.39 \\
1.99\end{array}$ & $\begin{array}{c}11.43 \pm 1.12 \\
0.67\end{array}$ \\
\hline
\end{tabular}

an integration time of $|T|=30$ frames. Only a single in situ medusa was used in the analysis due to difficulties in obtaining adequate sequences. Consequently, the in situ findings are informative, but should be considered preliminary.

\section{RESULTS}

\section{Capture efficiency measurements}

Prey tracks from laboratory data are used to illustrate the location of entrained or encountered prey relative to the Aequorea victoria's body (Fig. 2). Not all prey located upstream of the swimming animal were encountered (gray tracks), which is indicative of discrete fluid regions that are later entrained into the

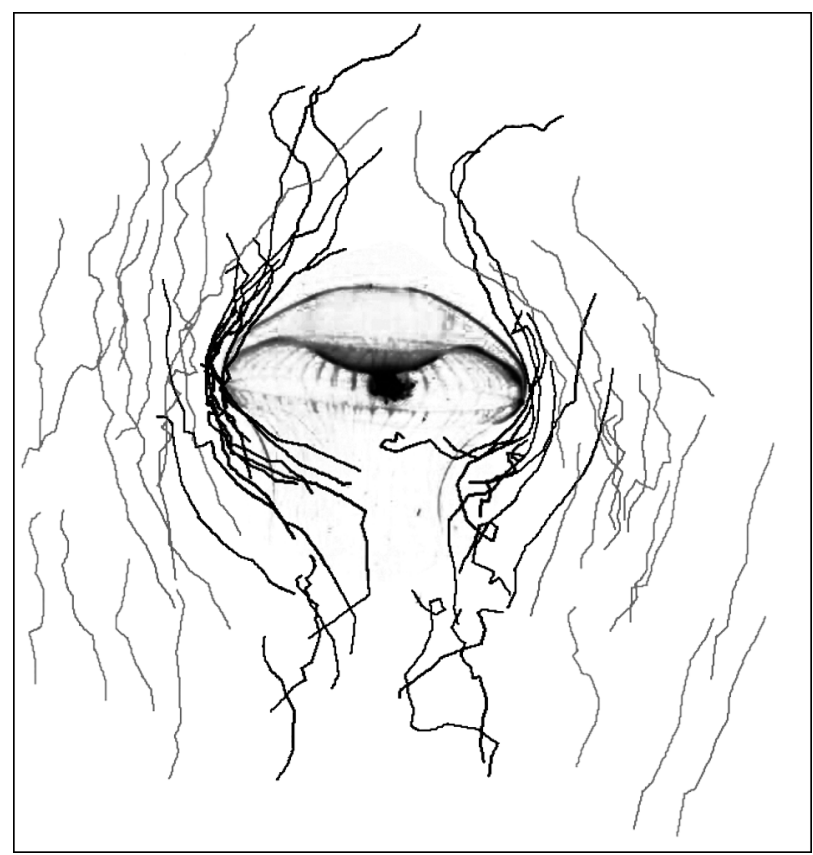

Fig. 2. Prey tracks around Aequorea victoria in the laboratory. Black tracks: prey that are entrained and encountered (or pass through tentacles); gray tracks: prey not encountered by the medusa animal's wake, confirming previous studies (Shadden et al. 2006, Peng \& Dabiri 2009). These discrete packets of fluid are transported into the vortex ring wake of the animal, which passes through downstream tentacles. Of those prey that were entrained or encountered, the number of prey that were captured by $A$. victoria's tentacles were then used to determine the capture efficiency (Fig. 3A). A. victoria captured the largest and slowest swimming prey most efficiently. Fast-swimming copepods were least vulnerable to capture.
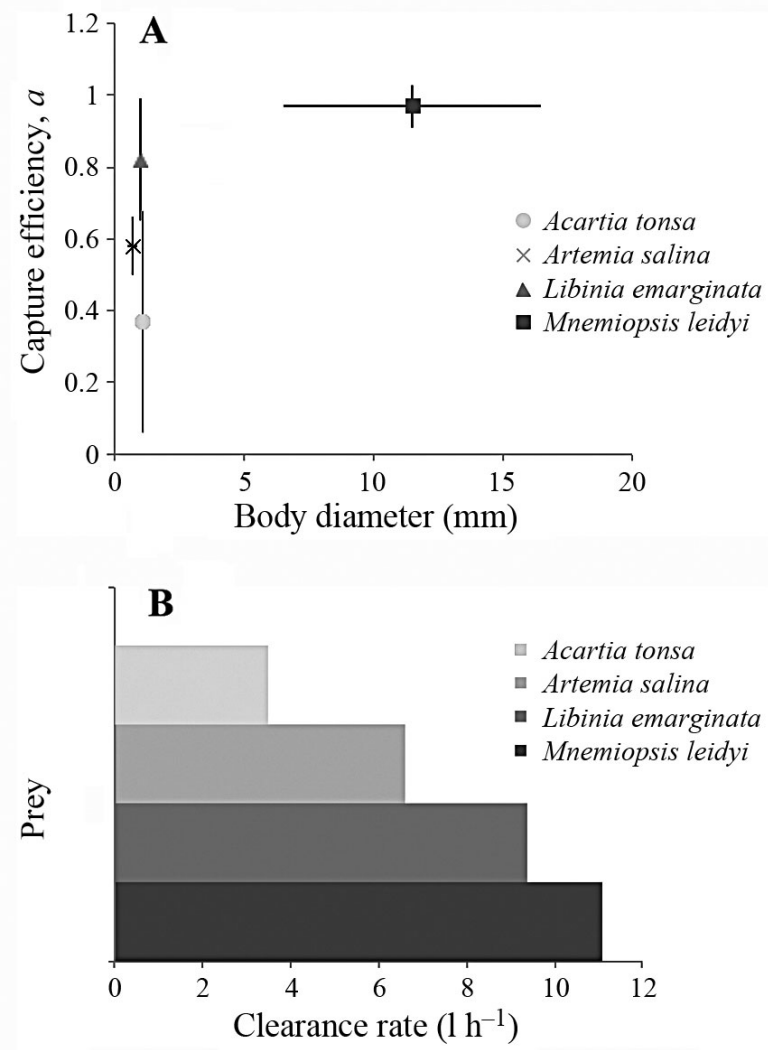

Fig. 3. (A) Prey capture efficiency and (B) clearance rate measurements for Aequorea victoria $(n=23)$ with different prey types. Clearance rate is estimated as the product of capture efficiency $(a)$ and encounter volume rate ( $E$; refer to Table 1$)$. Data for Libinia emarginata body sizes were acquired from Costello \& Henley (1971) 

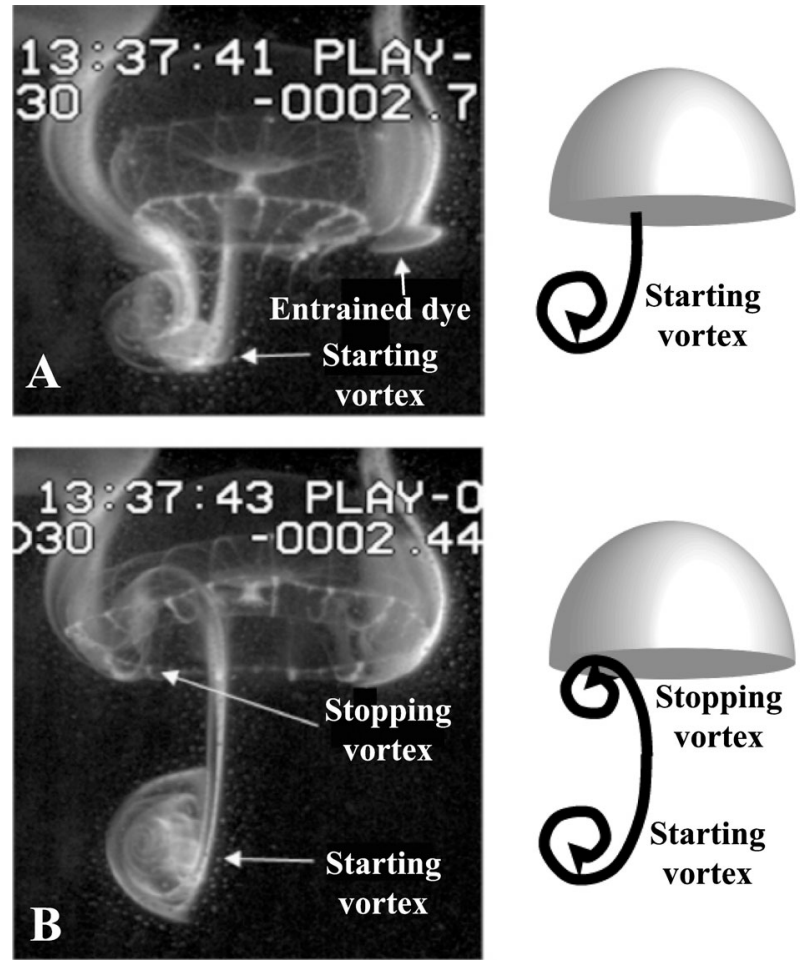

Fig. 4. Laboratory visualization of flow around Aequorea victoria using fluorescent dye. During bell contraction $\left(\mathrm{A}_{\text {; }}\right.$ maximum bell contraction shown) the starting vortex is formed and during expansion ( $\mathrm{B}_{\text {; }}$ maximum expansion shown) the stopping vortex is formed inside the subumbrellar cavity

\section{Flow visualization and DPIV}

During a single swimming cycle, 2 vortex ring structures can be visualized from the dye (see Fig. 4). During bell contraction (Fig. 4A), a starting vortex is formed in the wake of Aequorea victoria. During bell relaxation (Fig. 4B), a stopping vortex is formed inside the subumbrellar cavity. A time series of the velocity vector fields generated by a swimming $A$. victoria demonstrate that maximum fluid velocities are found within these vortices (indicated by white arrows) and areas adjacent to the bell margin (Fig. 5A). Shear fields calculated from the velocity vectors show that the vortices are also associated with the highest levels of shear around the swimming A. victoria (Fig. 6).
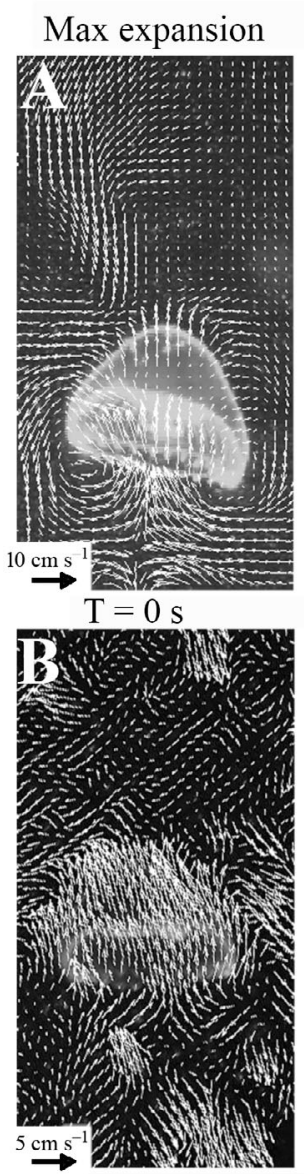

$\mathrm{T}=0 \mathrm{~s}$

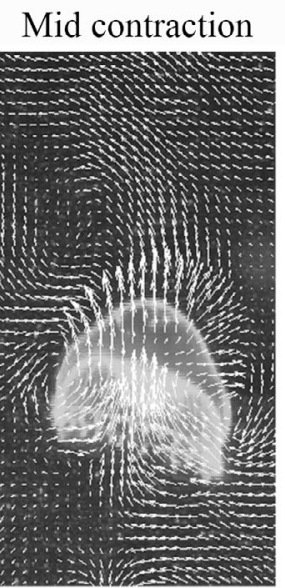

$0.17 \mathrm{~s}$

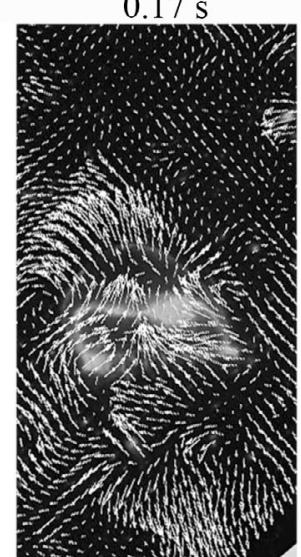

$0.2 \mathrm{~s}$
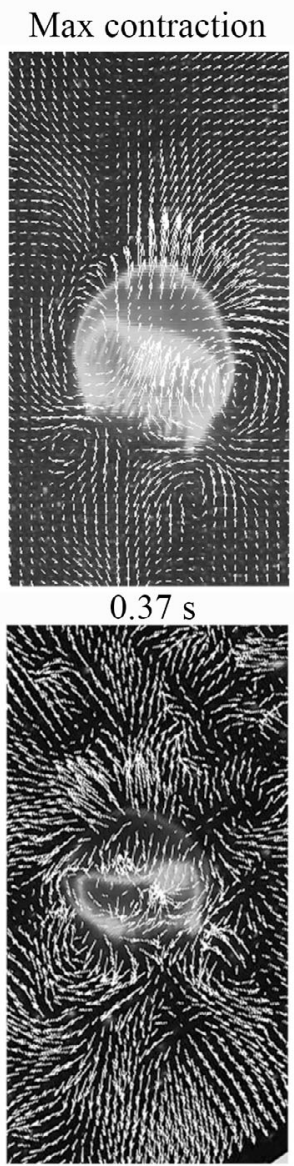

$0.37 \mathrm{~s}$

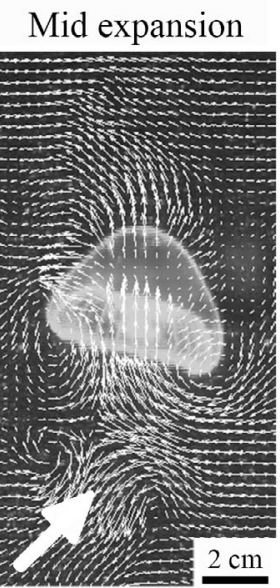

$0.53 \mathrm{~s}$

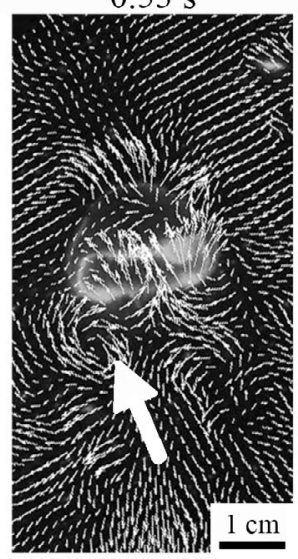

$0.57 \mathrm{~s}$
Fig. 5. Velocity vectors from digital particle image velocimetry (DPIV) analysis around swimming Aequorea victoria in (A) the laboratory and (B) the natural field setting. Large white arrows: vortex rings in the animal's wake 

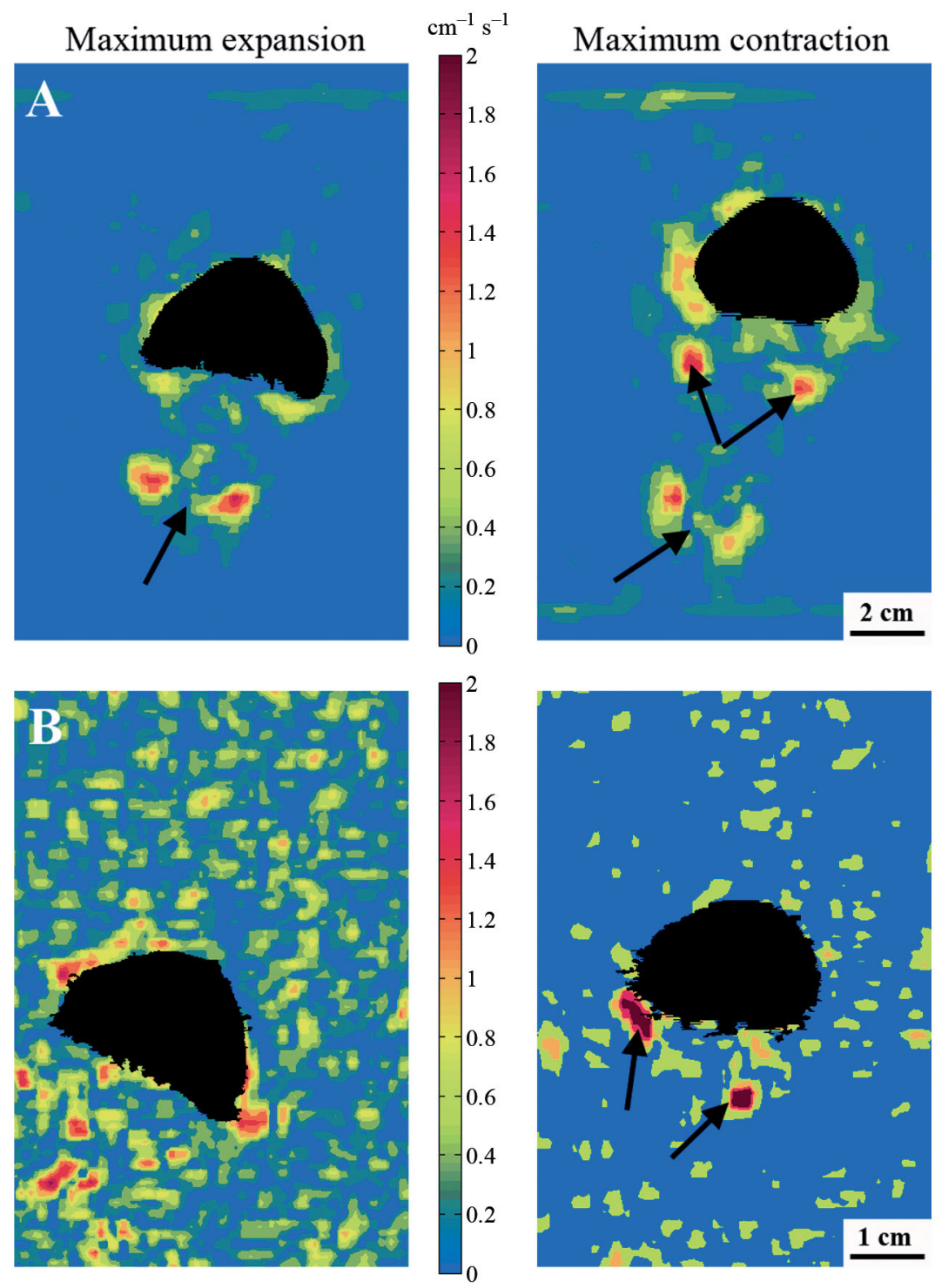

Fig. 6. Magnitude of total shear normalized by animal diameter $\left(\mathrm{cm}^{-1} \mathrm{~s}^{-1}\right)$ around Aequorea victoria swimming in (A) the laboratory and (B) the natural field setting at maximum expansion (left panels) and maximum contraction (right panels). Black arrows: vortex rings and vortex ring cores

The flow was measured around 4 other similarly sized Aequorea victoria, and the vector fields (not shown here) demonstrate that their fluid structures (i.e. generation of starting and stopping vortices) were similar to the medusae described in Figs. 4 \& $5 \mathrm{~A}$ and are summarized in Table 1. Maximum quantities in areas adjacent to the bell margin are included, since this region is where encounters with entrained prey are most often initiated (Costello \& Colin 1994, 1995, Ford et al. 1997). Fluid is entrained along the bell margin at maximum velocities of $6 \mathrm{~cm}$ $\mathrm{s}^{-1}$ and at shear rates of $0.50 \mathrm{~s}^{-1}$. These values varied little among replicate medusae $(n=5$, standard deviation near $10 \%$ ).

\section{Lagrangian analysis of flow}

The FTLE fields identify regions of convergent (attracting FTLE) and divergent (repelling FTLE) flow. Based on these regions we can identify discrete packets of fluid in front of the swimming medusa that will be encountered (i.e. transported through the capture surfaces). Fig. 7B illustrates how the FTLE fields identify convergent and divergent regions in the flow. Two representative FTLE ridges (indicated by black boxes in Fig. 7A) are identified as potential LCS because LCS correspond to ridges (or local maximums) in the FTLE field (Shadden et al. 2005). These potential LCS are redrawn relative to the animal body in Fig. 7B (red and blue curves). The red and blue dots identify actual particle pairs located on both sides of the attracting and repelling LCS, respectively. The position of these particle pairs is subsequently tracked through time (Fig. 7B). As predicted by the LCS, the position of particles on either side of an attracting or repelling LCS converges or diverges, respectively.

We identified regions of flow in front of the swimming Aequorea victoria by following the criteria for LCS extraction of a vortex ring (Shadden et al. 2006, Mathur et al. 2007), whereby the intersections of attracting and repelling ridges of FTLE fields yield the LCS that bounds the animal (identified by the black line; Fig. 7C). The fluid inside the LCS lobe structures located upstream of the swimming animal indicate finite regions of fluid that will be brought into the oral region of the medusa occupied by the tentacles (identified as 'encountered fluid'). Fig. $7 \mathrm{C}$ shows that only discrete regions of fluid in front of swimming $A$. victoria were entrained through these regions occupied by tentacles. The subsequent transport of the encountered fluid is tracked through time in the wake of the swimming medusa (red packets of particles). The motion of particles in the flow of swimming animals is similar to traditional particle-tracking techniques; however, we are able to predict regions of fluid that interact with the animal a priori due to the LCS analysis. As particles move, their position relative to the LCS remains the same (red markers remain inside and blue markers remain outside the LCS), since LCS are material lines (Shadden et al. 2005). In addition, we 


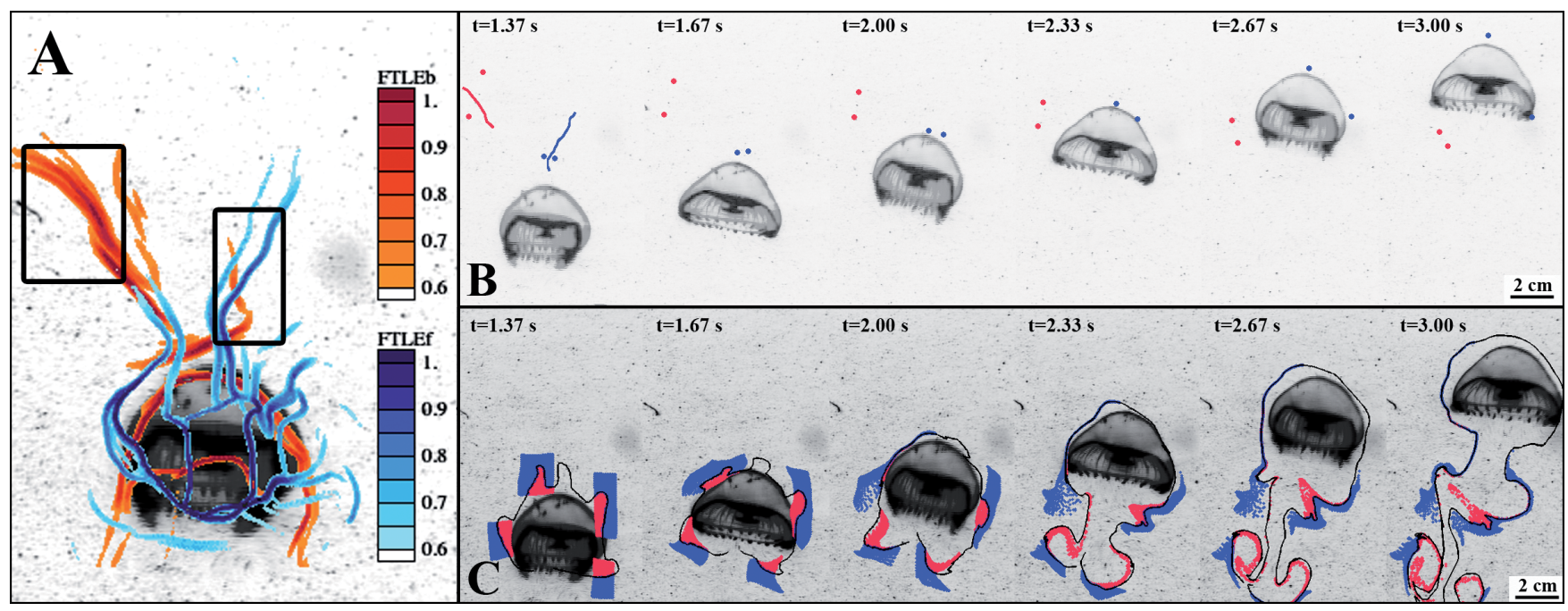

Fig. 7. (A) Laboratory finite-time Lyapunov exponent (FTLE) field surrounding Aequorea victoria computed using integration time $|T|=60$ frames of data. Black rectangles: location of potential attracting and repelling LCS shown in red and blue lines (in Panel B), respectively, at $t=1.37 \mathrm{~s}$. (B) Placement of particles adjacent to potential LCS illustrates the behavior of the flow. As time continues, particles adjacent to potential attracting LCS (indicated by red dots) will converge, and particles adjacent to potential repelling LCS (indicated by blue dots) will diverge. From the intersections and relative placement of attracting and repelling FTLE fields, the LCS surrounding the swimming animal can be found. (C) Particles located within the LCS (red) remain inside the LCS, and particles outside the LCS (blue) remain outside, thereby illustrating the material line property of LCS lobes

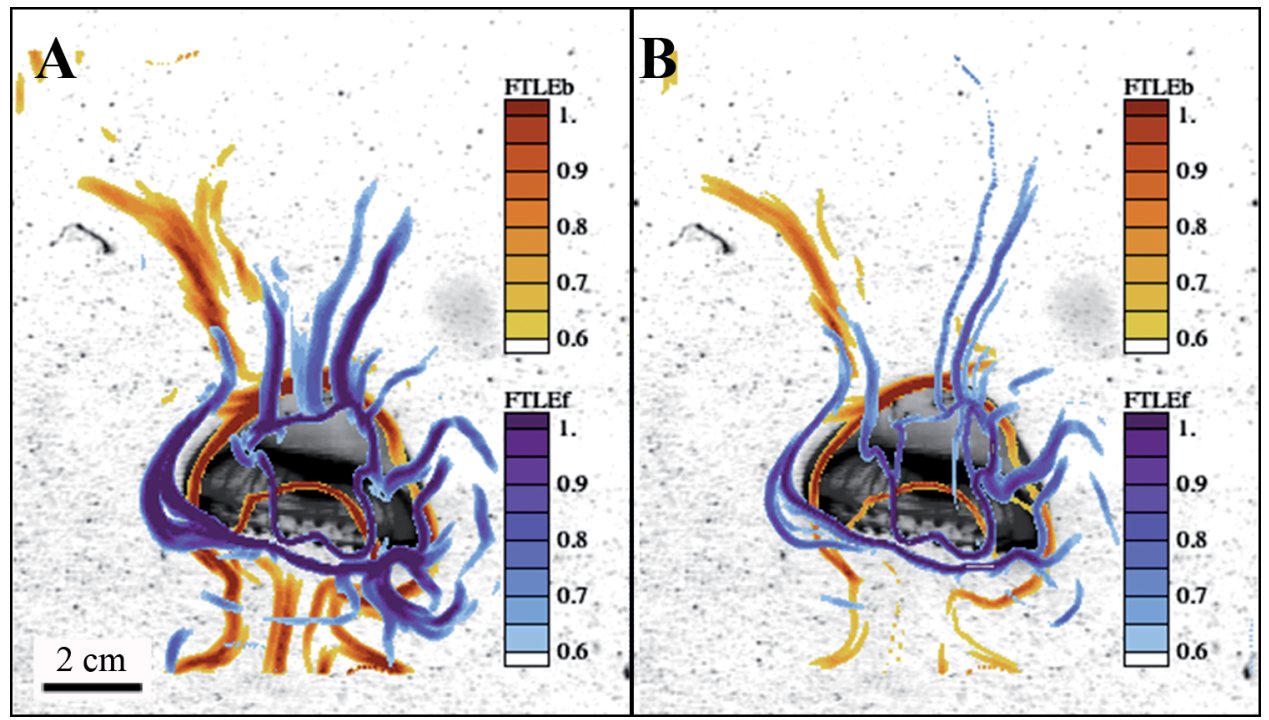

Fig. 8. FTLE fields surrounding Aequorea victoria in the laboratory using the same data set with integration time (A) $|T|=30$ and (B) $|T|=60$ frames. Blue and red contours correspond to repelling and attracting FTLE fields, respectively

see that particles located inside upstream lobes (red markers) are entrained into vortex rings that are generated in the animal's wake. Consequently, this enables us to use these LCS structures to determine the boundary of the fluid that is entrained and encountered by the medusa as it swims, enabling us to quantify the volume entrained.

To examine the effects of integration time $T$ on the LCS, we compared analyses based upon integration times of 30 and 60 frames of data (Fig. 8A and B, respectively) for the swimming sequence seen in Fig. 7. This is important because in some instances only a limited amount of video footage is available for analysis, thereby limiting the integration time (e.g. the in situ field observations in Fig. 9). The FTLE ridges adjacent to the animal body are more pronounced and numerous with longer integration times (Fig. 8B) than when using shorter integration times (Fig. 8A). Consequently, the number of upstream LCS lobes decreases as the integration time is reduced. These observations are consistent with previous published works (Romkedar et al. 1990, Shadden 2006). Despite these differences, the general structure of the flow remains the same as time moves forward. 
Fig. 9. (A) The position of an ambient attracting LCS (dashed black curve) relative to an LCS curve bound to the body of Aequorea victoria (solid black curve) starting at time $t=3.00 \mathrm{~s}$, with increments of $0.17 \mathrm{~s}$. (B) Extracted LCS surrounding the body of $A$. victoria (black curve) from the in situ FTLE field and the position of particles relative to the LCS curve as time progresses. Red and blue particles are initially placed inside and outside the upstream and downstream LCS lobes, respectively. A series of images starting at time $t=2.33$ $\mathrm{s}$, in increments of $0.5 \mathrm{~s}$, shows the evolution of particle position relative to the LCS

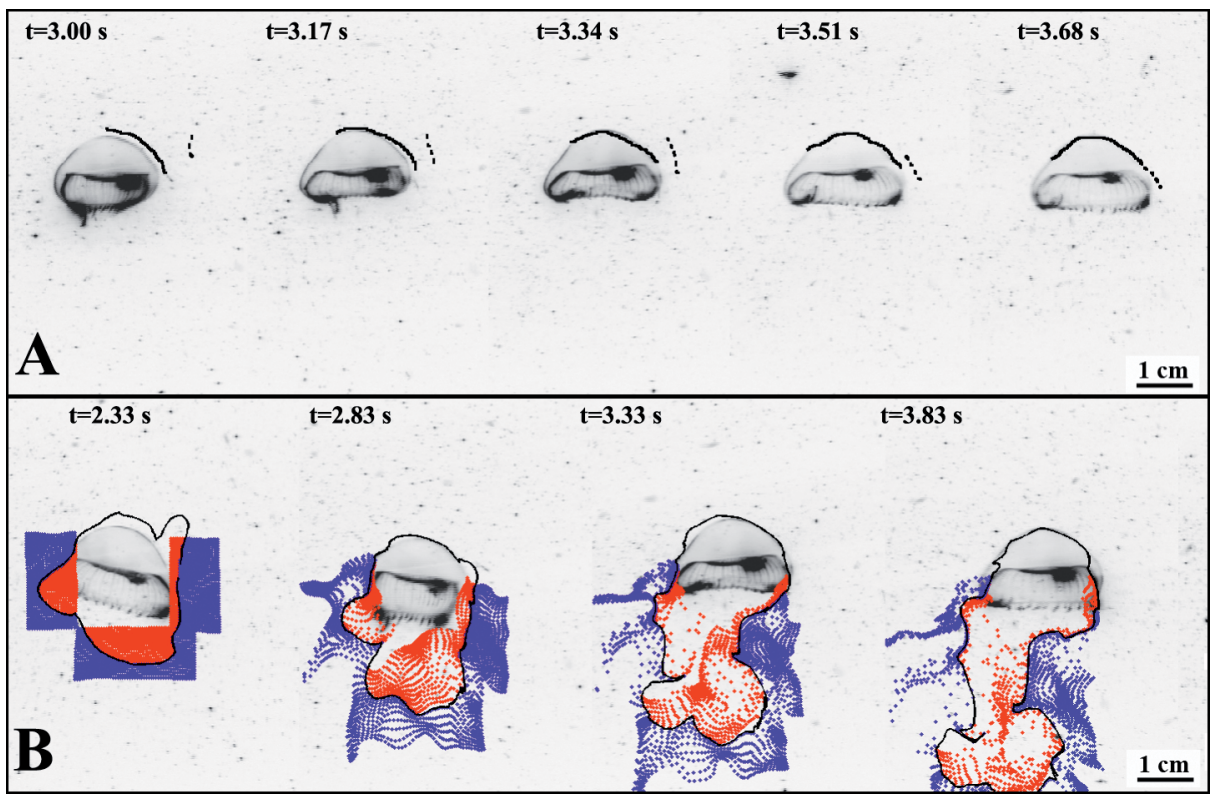

\section{Analysis of in situ fluid interactions}

Both the in situ velocity vector fields (Fig. 5B) and shear fields (Fig. 6B) reveal the complexity of ambient flow surrounding medusae in natural field settings. In order to estimate how these ambient flow structures affect fluid transport by medusae, we collected a sequence of continuous swimming in the field to analyze the video for FTLE contours. However, the data collected are only sufficient for LCS analysis at shorter integration times ( $|T|=30$ frames). As mentioned previously, shorter integration times may produce a less refined picture of LCS curves than longer integration times. Nonetheless, the analysis is informative for illustrating the influence of ambient flows on the structure of the flows around swimming medusae. We see a strong interaction of maximal FTLE contours adjacent to the animal (solid black curve) and maximal FTLE contours in the ambient fluid (dashed black curve) (Fig. 9A). As time moves forward, the LCS found in the ambient fluid later attaches to the LCS that bounds the animal body. Physically, this means that the ambient flow generates regions of strong particle separation and attraction independent of Aequorea victoria's feeding currents. As an animal swims and encounters background flow in the ambient fluid environment, it interacts with these flow structures, which results in the combining or separating of LCS from the animal body.

The presence of active flow structures in the surrounding fluid and their interaction with swimming medusae likely result in altered fluid transport compared to still water in filming vessels. Using the same LCS extraction criteria as before, the intersections of attracting and repelling ridges of FTLE fields yield the
LCS (black curve) that bounds the animal but excludes the LCS in the ambient flow (Fig. 9B at $t=2.33 \mathrm{~s}$ ). Red and blue markers are initially placed inside and outside upstream and downstream LCS lobes, respectively. As in the laboratory case, red and blue particles remain inside and outside the bounding LCS, respectively. However, unlike the laboratory case (Fig. 7C), particles initially located inside the upstream LCS lobes (red markers) in the natural field setting are not entirely entrained in the vortex rings present in the animal wake. Consequently, the amount of fluid that is transported into the capture region is greatly reduced (Table 1).

\section{DISCUSSION}

\section{Flow and the encounter process}

Observed clearance rates of predators are determined by the rate at which prey are encountered (quantified as the encounter volume rate, $E$ ) and the efficiency with which encountered prey are captured and retained for ingestion (quantified as the capture efficiency, $a$, the number of ingested prey/number of encountered prey; Kiørboe 2011). For feeding current predators, such as oblate medusae, which transport fluid through capture surfaces (e.g. tentacles) and filter out prey, the encounter rate kernel can be approximated as $E=A v$, where $A$ is the area of the filter (i.e. tentacles) and $V$ is the velocity of the fluid (Kiørboe 2011). Unfortunately, as a result of the dynamic nature of trailing tentacles and the complexity of the fluid velocities in the wake of swimming medusae, there 
have been very few attempts to estimate $E$ for feeding current-foraging medusae (exceptions include Madin 1988). However, laboratory-based LCS analysis enabled us to identify structures in the flow and determine how much fluid is transported through the tentacles of Aequorea victoria. The volume of the LCS lobe divided by the swimming cycle duration provided an estimate of the encounter volume rate, E. Accordingly, in the laboratory, A. victoria transported fluid through the tentacles at a rate of $11.4 \mathrm{l} \mathrm{h}^{-1}$ (Table 1). This estimate of $E$ is conservative, since it only considers the transport proximate to the bell and tentacles can extend beyond this region. Despite this, our estimates of $E$ are on the same order as the previously published clearance rates for A. victoria (Purcell 2003) and for other species of comparably sized oblate medusae (Titelman \& Hansson 2005).

In addition to providing flow-based estimates of encounter rates, the LCS analysis (Fig. 7C) and the prey transport tracks (Fig. 2) demonstrate the complex nature of how Aequorea victoria interacts with its surrounding fluid. Not surprisingly, the prey tracks and the LCS confirm that $A$. victoria, like other oblate medusae, transports fluid from in front of its bell, along the regions immediately adjacent to the bell margin, and circulates the fluid into the trailing wake. However, unlike previous suggestions, it does not encounter a simple cylinder of fluid estimated as the product of the cross-sectional area of the bell and the swimming velocity (e.g. Bailey \& Batty 1983 for Aurelia aurita). Instead, Ae. victoria entrains only discrete packets of fluid through its tentacles, and only a portion of the prey located upstream of a swimming medusae are actually encountered (Fig. 2). In fact, Ae. victoria only entrained about $30 \%$ of the Bailey \& Batty (1983) cylinder, creating a mosaic of fluid that is encountered (indicated by upstream LCS lobes). Consequently, more quantitative, flow-based methods, such as LCS analysis are required to achieve accurate estimates of $E$ for oblate feeding current-foraging medusae.

The characteristics we describe of how Aequorea victoria interacts with its surrounding fluid in a laboratory environment are similar to those described for the oblate scyphomedusa Aurelia aurita using a similar LCS analysis (Shadden et al. 2006, Peng \& Dabiri 2009). These similarities between Ae. victoria, a hydromedusa, and Au. aurita, a scyphomedusa, support the concept that feeding current-foraging medusae transport fluid similarly, regardless of their evolutionary origins (Colin et al. 2006, Costello et al. 2008). Consequently, the processes governing the initial encounters with prey are similar. However, encounter rates and capture efficiencies also depend upon the morphology of capture surfaces (such as tentacles and oral arms), and these differ greatly among rowing medusae.

\section{Post-encounter interactions}

As mentioned, clearance rates are also a function of the efficiency with which encountered prey are retained for ingestion. Specifically, ingestion requires that entrained prey be transported and contact capture surfaces without escaping. Based on measured capture efficiencies, $a$, we found that Aequorea victoria was most effective at retaining large, slow, soft-bodied prey. The high capture efficiencies observed for eggs and gelatinous prey are consistent with known feeding patterns in the field (Purcell \& Mills 1988, Purcell 1991, Mills 1995, Costello \& Colin 2002). The product of $E$ from the laboratory LCS analysis and a from predatorprey interactions provide estimates of clearance rates of different prey types in non-limiting food conditions (Fig. 3B). These values are consistent with clearance rates measured from gut content data. For example, Aequorea aequorea cleared fish larvae at about $12 \mathrm{l} \mathrm{h}^{-1}$ (Purcell \& Arai 2001).

Previous studies suggest that oblate medusae, which use feeding currents, should not be capable of entraining and encountering copepod prey because copepods should be able to detect and escape from their feeding currents (Costello \& Colin 1994, 1995). The more quantitative data obtained using DPIV demonstrate that the maximum marginal flow velocities of Aequorea victoria were 2-fold greater than those observed in previous studies of other similarly sized medusae (Costello \& Colin 1994, Colin \& Costello 2002). This has important implications for feeding as marginal flow velocities are thought to be an important parameter in determining prey selection by oblate medusae, because prey with slower escape speeds than the entraining flow are more likely to be captured than prey with faster escaping speeds such as copepods (Costello \& Colin 1994). For example, our observed marginal flow velocities were below the escape velocities of some copepods (e.g. Burdick et al. 2007, Waggett \& Buskey 2007), but greater than others (e.g. Suchman \& Sullivan 1998, Viitasalo et al. 1998). Further, the levels of fluid shear observed along the bell margin were below the deformation rates that elicit escape responses from several copepods (Table 2), suggesting that many copepods can be entrained into capture zones before detecting a threat. However, shear levels in the vortices rotating through the tentacles reach levels at or above many copepod thresholds. Therefore, it would be possible for copepods to be entrained in medusan feeding currents, but detect the predator and attempt an escape before contacting a tentacle (Kiørboe et al. 1999, Green et al. 2003, Burdick et al. 2007). However, the strength of vortices generated in the wake of A. victoria, and other rowing medusae, may prevent escape of prey in encountered fluid regions as they pass 
Table 2. Threshold deformation rates of selected species of copepods measured in the laboratory

\begin{tabular}{|lcl|}
\hline Prey species & $\begin{array}{c}\text { Deformation } \\
\text { rate }\left(\mathrm{s}^{-1}\right)\end{array}$ & Source \\
\hline Acartia tonsa & 0.34 & Kiørboe et al. (1999) \\
Calanus finmarchicus & 0.4 & Haury et al. (1980) \\
Temora longicornis & 6.5 & Kiørboe et al. (1999) \\
Eurytemora affinis & 1.9 & Viitasalo et al. (1998) \\
Euchaeta rimana & 2.4 & Fields \& Yen (1997) \\
Oithona sp. & 3.8 & Kiørboe et al. (1999) \\
\hline
\end{tabular}

through trailing tentacles, improving encounter rates. These findings suggest that, due to greater encounter rates, copepods may be more vulnerable to predation by some cruising-foraging medusae than previously thought. This is consistent with observations of interactions between copepods and some feeding current-foraging medusae (Suchman \& Sullivan 1998, 2000 for Chrysaora quinquecirrha) and some gut content data (Sullivan et al. 1994, Barz \& Hirsch 2005 for Aurelia aurita). However, as mentioned, prey selection is also a function of capture efficiencies, which are largely determined by nematocyst arrays in medusae (Purcell \& Mills 1988). The nematocyst types observed for $A$. victoria are effective at capturing soft-bodied prey and not crustaceans (Purcell \& Mills 1988). Therefore, while copepods may be encountered by A. victoria, our capture efficiency data and in situ observations suggest that $A$. victoria is not effective at capturing copepods (Purcell \& Mills 1988, Purcell 1991, Mills 1995, Costello \& Colin 2002).

\section{Relevance of laboratory observations in the field}

It is difficult to evaluate the relevance of laboratorybased observations and predictions for natural field settings. Our field data are limited to a single animal, yet provide insight into how flow around foraging medusae may be influenced by natural flow conditions. The presence of background flow is highly apparent in the in situ velocity and shear fields (Figs. 5B \& 6B). This flow appears to distort and redistribute upstream LCS lobes unevenly on either side of the animal, creating asymmetric flows and fluid transport (Fig. 9B). In addition, vortex ring structures in the wake of the in situ Aequorea victoria lack coherent shape. As a result, the size of the LCS lobes entrained by the medusae is reduced by $50 \%$ (occupying 14 versus $30 \%$ of the cylinder of fluid through which the bell interacts). However, during calm periods in the natural field setting, background flow structures dissipate, revealing similar vortical wake structures observed in the laboratory, but with a slightly weaker signal (Fig. 5B, $t=0.57 \mathrm{~s}$ ). These effects on fluid structure shape and location suggest that medusae may have less control over fluid and prey transport in a natural field setting than under quiescent flows in the laboratory. However, the overall effect on encounter is difficult to predict, since turbulence is known to enhance predator-prey encounter rates (MacKenzie \& Kiørboe 1995). Further, high shear in ambient flow is of the same order of magnitude as shear generated by a swimming predator (Fig. 6B). This may make the medusa less detectable to prey and, hence, the prey more vulnerable to predation. Consequently, while the in situ data are currently limited, LCS analysis with in situ data does provide an approach for future studies by demonstrating that ambient flows may alter patterns of fluid entrainment by medusae and influence the detection of medusan predators by their prey.

\section{CONCLUSIONS}

DPIV analysis demonstrates that the feeding current generated by Aequorea victoria entrains packets of fluid and may be strongly influenced by ambient flows. The use of DPIV vector fields to measure the Lagrangian flow around swimming medusae enables us to predict the encounter volume rate of predators. This is a powerful tool that can be used to evaluate how different morphologies and swimming behaviors may impact the feeding rates of predators which use feeding currents to encounter prey.

Acknowledgements. This research is supported by the National Science Foundation awarded to J.O.D. (OCE-0623475), S.P.C. (OCE-0623534 and OCE-0727544) and J.H.C. (OCE0351398 and OCE-0623534) and by the Office of Naval Research awarded to J.H.C. (N000140810654). K.K.Y. was supported by a National Defense Science and Engineering Graduate Fellowship.

\section{LITERATURE CITED}

Adrian RJ (1991) Particle-imaging techniques for experimental fluid mechanics. Annu Rev Fluid Mech 23:261-304

Bailey KM, Batty RS (1983) A laboratory study of predation by Aurelia aurita on larval herring (Clupea harengus): experimental observations compared with model predictions. Mar Biol 72:295-301

Barz K, Hirsch HJ (2005) Seasonal development of scyphozoan medusae and the predatory impact of Aurelia aurita on the zooplankton community in the Bornholm Basin (central Baltic Sea). Mar Biol 147:465-476

Batchelor GK (1967) An introduction to fluid dynamics. Cambridge University Press, Cambridge

> Burdick DS, Hartline DK, Lenz PH (2007) Escape strategies in co-occurring calanoid copepods. Limnol Oceanogr 52: 2373-2385 
Buskey EJ, Lenz PH, Hartline DK (2002) Escape behavior of planktonic copepods in response to hydrodynamic disturbances: high speed video analysis. Mar Ecol Prog Ser 235: 135-146

> Colin SP, Costello JH (2002) Morphology, swimming performance and propulsive mode of six co-occurring hydromedusae. J Exp Biol 205:427-437

Colin SP, Costello JH, Kordula H (2006) Upstream foraging by medusae. Mar Ecol Prog Ser 327:143-155

> Costello JH, Colin SP (1994) Morphology, fluid motion and predation by the scyphomedusa Aurelia aurita. Mar Biol 121:327-334

> Costello JH, Colin SP (1995) Flow and feeding by swimming scyphomedusae. Mar Biol 124:399-406

> Costello JH, Colin SP (2002) Prey resource utilization by cooccurring hydromedusae from Friday Harbor, Washington, USA. Limnol Oceanogr 47:934-942

Costello DP, Henley C (1971) Methods for obtaining and handling marine eggs and embryos. Marine Biological Laboratory, Woods Hole, MA

Costello JH, Colin SP, Dabiri JO (2008) Constraints and consequences in medusan evolution. Invertebr Biol 127: 265-290

Dabiri JO, Gharib M (2004) Fluid entrainment by isolated vortex rings. J Fluid Mech 511:311-331

> Dabiri JO, Colin SP, Costello JH, Gharib M (2005) Flow patterns generated by oblate medusan jellyfish: field measurements and laboratory analyses. J Exp Biol 208: 1257-1265

> Dabiri JO, Colin SP, Katija K, Costello JH (2010) A wakebased correlate of swimming performance and foraging behavior in seven co-occurring jellyfish species. J Exp Biol 213:1217-1225

Fields DM, Yen J (1997) The escape behavior of marine copepods in responds to a quantifiable fluid mechanical disturbance. J Plankt Res 19(9):1289-1304

Ford MD, Costello JH, Klos E (1997) Swimming and feeding by the scyphomedusa Chrysaora quinquecirrha. Mar Biol 129:355-362

Franco E, Pekarek DN, Peng J, Dabiri JO (2007) Geometry of unsteady fluid transport during fluid-structure interactions. J Fluid Mech 589:125-145

Gerritsen J, Strickler J (1977) Encounter probabilities and community structure in zooplankton: a mathematical model. J Fish Res Board Can 34:73-82

Green S, Visser AW, Titelman J, Kiørboe T (2003) Escape responses of copepod nauplii in the flow field of the blue mussel, Mytilus edulis. Mar Biol 142:737-742

Green MA, Rowley CW, Haller G (2007) Detection of Lagrangian coherent structures in three-dimensional turbulence. J Fluid Mech 572:111-120

Haller G (2001) Lagrangian structures and the rate of strain in a partition of two-dimensional turbulence. Phys Fluids 13: 3365-3385

> Haller G (2002) Lagrangian coherent structures from approximate velocity data. Phys Fluids 14:1851-1861

Haury LR, Kenyon DE, Brooks JR (1980) Experimental evaluation of the avoidance reaction of Calanus finmarchicus. J Plankton Res 2(3):187-202

Katija K, Dabiri JO (2008) In situ field measurements of aquatic animal-fluid interactions using a self-contained underwater velocimetry apparatus (SCUVA). Limnol Oceanogr Methods 6:162-171

Kiørboe T (2011) How zooplankton feed: mechanisms, traits and trade-offs. Biol Rev Camb Philos Soc 86:311-339
Kiørboe T, Saiz E, Visser A (1999) Hydrodynamic signal perception in the copepod Acartia tonsa. Mar Ecol Prog Ser 179:97-111

> MacKenzie BR, Kiørboe T (1995) Encounter rates and swimming behavior of pause-travel and cruise larval fish predators in calm and turbulent laboratory experiments. Limnol Oceanogr 40:1278-1289

Madin LP (1988) Feeding behavior of tentaculate predators: in situ observations and a conceptual model. Bull Mar Sci 43:413-429

Mathur M, Haller G, Peacock T, Ruppert-Felsot JE, Swinney HL (2007) Uncovering the Lagrangian skeleton of turbulence. Phys Rev Lett 98:144502

Matsakis S, Conover RJ (1991) Abundance and feeding of medusae and their potential impact as predators on other zooplankton in Bedford Basin (Nova Scotia, Canada) during spring. Can J Fish Aquat Sci 48:1419-1430

Mills CE (1981) Diversity of swimming behaviors in hydromedusae as related to feeding and utilization of space. Mar Biol 64:185-189

Mills CE (1995) Medusae, siphonophores, and ctenophores as planktivorous predators in changing global ecosystems. ICES J Mar Sci 52:575-581

Peng J, Dabiri JO (2007) A potential-flow, deformable-body model for fluid-structure interactions with compact vorticity: application to animal swimming measurements. Exp Fluids 43:655-664

> Peng J, Dabiri JO (2009) Transport of inertial particles by Lagrangian coherent structures: application to predatorprey interactions in jellyfish feeding. J Fluid Mech 623: 75-84

Purcell JE (1989) Predation by the hydromedusa Aequorea victoria on fish larvae and eggs at a herring spawning ground in British Columbia. Can J Fish Aquat Sci 46: 1415-1427

> Purcell JE (1991) Predation by Aequorea victoria on other species of potentially competing pelagic hydrozoans. Mar Ecol Prog Ser 72:255-260

Purcell JE (2003) Predation on zooplankton by large jellyfish, Aurelia labiata, Cyanea capillata and Aequorea aequorea, in Prince William Sound, Alaska. Mar Ecol Prog Ser 246: 137-152

Purcell JE, Arai MN (2001) Interactions of pelagic cnidarians and ctenophores with fish: a review. Hydrobiol 451:27-44

> Purcell JE, Cowan JH Jr (1995) Predation by the scyphomedusan Chrysaora quinquecirrha on Mnemiopsis leidyi ctenophores. Mar Ecol Prog Ser 129:63-70

Purcell JE, Mills CE (1998) The correlation between nematocyst types and diets in pelagic hydrozoa. In Hessinger DA, Lenhoff $\mathrm{H}$ (eds). The biology of nematocysts. Academic Press, New York, NY

Raskoff KA (2002) Foraging, prey capture, and gut contents of the mesopelagic narcomedusae Solmissus sp. (Cnidaria: Hydrozoa). Mar Biol 141:1099-1107

> Romkedar V, Leonard A, Wiggins S (1990) An analytical study of transport, mixing and chaos in an unsteady vortical flow. J Fluid Mech 214:347-394

Shadden SC (2006) A dynamical systems approach to unsteady systems. PhD thesis, California Institute of Technology, Pasadena, CA

> Shadden SC, Lekien F, Marsden JE (2005) Definition and properties of Lagrangian coherent structures from finitetime Lyapunov exponents in two-dimensional aperiodic flows. Physica D 212:271-304

> Shadden SC, Dabiri JO, Marsden JE (2006) Lagrangian 
analysis of fluid transport in empirical vortex ring flows. Phys Fluids 18:047105-047111

Suchman CL, Sullivan BK (1998) Vulnerability of the copepod Acartia tonsa to predation by the scyphomedusa, Chrysaora quinquecirrha. Mar Biol 132:237-245

Suchman CL, Sullivan BK (2000) Effect of prey size on vulnerability of copepods to predation by the scyphomedusae Aurelia aurita and Cyanea sp. J Plankton Res 22: 2289-2306

Sullivan BK, Garcia JR, Klein-MacPhee G (1994) Prey selection by the scyphomedusan predator Aurelia aurita. Mar Biol 121:335-341

Titelman J, Hansson LJ (2005) Feeding rates of the jellyfish Aurelia aurita on fish larvae. Mar Biol 149:297-306

Editorial responsibility: Marsh Youngbluth,

Fort Pierce, Florida, USA
Tytell ED, Lauder GV (2004) The hydrodynamics of eel swimming. I. Wake structure. J Exp Biol 207:1825-1841

Videler JJ, Stamhuis EJ, Muller UK, van Duren LA (2002) The scaling and structure of aquatic animal wakes. Integr Comp Biol 42:988-996

Viitasalo M, Kiørboe T, Flinkman J, Pedersen LW, Visser AW (1998) Predation vulnerability of planktonic copepods: consequences of predator foraging strategies and prey sensory abilities. Mar Ecol Prog Ser 175:129-142

Waggett RJ, Buskey EJ (2007) Calanoid copepod escape behavior in response to a visual predator. Mar Biol 150: 599-607

Willert CE, Gharib M (1991) Digital particle image velocimetry. Exp Fluids 10:181-193

Submitted: January 18, 2011; Accepted: May 13, 2011 Proofs received from author(s): August 11, 2011 